\title{
Comparative evaluation of indices and partial-mouth periodontal protocols for epidemiological surveys
}

\begin{abstract}
Objective: To compare the Community Periodontal Index (CPI), the CPI modified and three partial-mouth periodontal protocols for estimates of prevalence, severity and extent of periodontitis in populations.

Method: a convenience sample of 350 individuals (aged 35 to 74 years) from Sao Paulo underwent a full-mouth periodontal examination (FMPE) which assessed pocket depth (PD), clinical attachment level (CAL) and bleeding on probing on six sites per tooth. The CPI, CPI modified (CPIm) and three partial-mouth protocols examination (PMPE)-halfmouth 6 sites, full- and half-mouth 3 sites-were derived from the records of the FMPE and have been compared for sensitivity, absolute bias, relative bias and inflation factor in estimates of periodontitis.
\end{abstract}

Results: Significant differences were found in periodontitis prevalence estimates between PMPE, in different case definitions, with relative biases ranging from $-10 \%$ to $-55 \%$. The CPIm had sensitivity of $100 \%$ for gingivitis and PD $\geq 4 \mathrm{~mm}$ prevalence, and $80 \%$ for moderate and severe periodontitis in relation to FMPE, while for CPI such estimates were $70 \%$ and $50 \%$, respectively. The full-mouth 3 sites protocol was similar to the CPIm, regarding the prevalence estimate, but CPIm overestimated severity and extent of periodontitis. The random half-mouth protocols presented low sensitivity to estimate periodontitis prevalence, although they presented small biases for severity and extension $(<2.0 \%)$

Conclusion: The CPIm and the full-mouth 3 sites protocol presented satisfactory sensitivity to estimate prevalence of periodontitis in populations, being superior to the previous CPI and to the random half-mouth protocols. However, accuracy of estimates may vary with the case definition and population characteristics.

Keywords: periodontitis, periodontal indices, prevalence, epidemiological surveillance
Volume II Issue 4 - 2020

\author{
Fernanda Cristina Figueira Teixeira,' Leticia \\ Marin-León,' Everton Padilha Gomes, ${ }^{2}$ Alice \\ Moreira Neves Pedrao, ${ }^{3}$ Alexandre da Costa \\ Pereira, ${ }^{2}$ Priscila Maria Stolses Bergamo \\ Francisco \\ 'Department of Public Health, Campinas State University, Brazil \\ ${ }^{2}$ School of Medicine, Health Institute, University of Sao Paulo, \\ Brazil \\ ${ }^{3}$ City Hall of Embu Guaçu, Sao Paulo, Brazil
}

Correspondence: Fernanda Cristina Figueira Teixeira, Department of Public Health, Campinas State University, Brazil, Tel (19) 3858-1435, Email fernandafigueirateixeira@gmail.com

Received: July 02, 2020 | Published: July 232020
Abbreviations: FMPE, full-mouth periodontal examination protocol; CPI, community periodontal index; CAL, clinical periodontal attachment loss; PD, probing depth; PMPE, partial-mouth periodontal examination protocol

\section{Introduction}

Epidemiological surveillance of periodontal disease is fundamental for identifying people at risk in the population, establishing preventive and therapeutic strategies and assessing their efficacy, as well as assessing the interactions between periodontitis and systemic diseases and their impact on quality of life. ${ }^{1,2}$ However, the lack of uniform criteria for assessing periodontitis in populations and the considerable methodological variability in examination protocols and case definitions lead to difficulties with regard to the interpretation and comparability of findings from different studies. ${ }^{3,4}$

The optimal periodontal examination consists of a full-mouth assessment, in which the probing depth and clinical attachment level are obtained from six sites per tooth, resulting in a large amount of scores per individual. The logistics, execution time, costs and fatiguing work required for the full-mouth periodontal examination protocol (FMPE) make it impractical for large epidemiological surveys. ${ }^{5}$ Consequently, several partial-mouth periodontal examination protocols (PMPE) have been proposed to assess periodontitis in populations, which vary with regard to the selection of specific teeth, sites or quadrants in the dental arch, often leading to biases.

Several studies have been carried out to evaluate accuracy of partial-mouth periodontal protocols. ${ }^{7}$ A recent consensus by the EU/
USA Periodontal Epidemiology Working Group ${ }^{8}$ recommended three PMPEs for estimates of periodontitis in populations: the fullmouth 3-site protocol and the two quadrants (half mouth) protocols of 3 and 6 sites. On the other hand, the method recommended by the World Health Organization (WHO), widely used worldwide, is the Community Periodontal Index (CPI) ${ }^{9}$ which is a simple, quick and universal test, however it has some limitations that may compromise its accuracy. ${ }^{3,7,10,11}$ The current version of the CPI (named here as CPIm) presents a modification in the examination protocol, with assessment of probing depth in all the present teeth, instead of only in ten index teeth. ${ }^{12}$ However, no studies have been identified that have evaluated the accuracy of CPIm in relation to the FMPE and other partial protocols.

The aim of the present study was to compare the Community Periodontal Index (CPI), the CPI modified and three partial-mouth periodontal protocols for estimates of prevalence, severity and extent of periodontitis in populations.

\section{Method}

A cross-sectional study was conducted involving an analysis of the clinical periodontal parameters of adult individuals (35 to 74 years) selected by convenience from a major study named Advento, ${ }^{13}$ whose participants were from the metropolitan region of Sao Paulo, Brazil. The invitation to participate in an oral assessment was made by telephone and considered volunteers who had been included in the chronogram of examinations in the Advento Study in the previous six months and had at least four natural teeth present (not including third 
molars). Individuals that had been submitted to periodontal treatment in the previous six months and those who had used antibiotics in the previous 30 days were excluded from the study. Based on these criteria, among the 586 eligible volunteers, those who consented to participate and appeared for the oral examination were included in this study $(\mathrm{n}=350)$.

After a previous interview, the participants were examined seated in a common chair, by four trained examiners that have been previously calibrated in the assessment of periodontal parameters. The level of inter-examiner agreement regarding the occurrence of periodontal pocket and bleeding on probing was above $85 \%$. Reproducibility in measures of clinical periodontal attachment loss (CAL) and probing depth (PD) was verified using the Kappa coefficient, which ranged from 0.68 to 0.86 .

A full-mouth periodontal examination protocol (FMPE) was performed at six sites per tooth (three buccal sites and three lingual sites). A standard millimeter probe (WHO model, with $0.5 \mathrm{~mm}$ sphere) and a dental mirror were used to assess the following periodontal variables: probing depth of sulcus/pocket (PD), defined as the distance between the free gingival margin and base of the sulcus/pocket in millimeters; clinical attachment loss (CAL), defined as the distance between the cemento-enamel junction and base of the sulcus/pocket in millimeters; bleeding on probing (BOP), checked approximately 10 seconds after probing. The measurements of PD and CAL were rounded down to the nearest whole millimeter. It was considered the occurrence of one or more bleeding sites for estimate prevalence of BOP.

The data for the PMPEs were obtained based on the records of the FMPE in accordance with the characterization of each protocol, as shown in Table 1. The randomization of quadrants, when necessary, was done manually, by drawing cards numbered 1 to 4 .

The variables evaluated were: BOP (at least one site); periodontal pocket $(\mathrm{PD} \geq 4 \mathrm{~mm})$; attachment loss $(\mathrm{CAL} \geq 4 \mathrm{~mm})$; mean $\mathrm{PD}$ and mean CAL for each participant and for the whole set, in millimeters (severity of periodontitis); mean frequencies of PD and CAL in different levels $(<4 \mathrm{~mm}, \geq 4 \mathrm{~mm}$ and $\geq 6 \mathrm{~mm}$ ), given by the proportion of affected teeth and/or sites in the total of teeth and/or sites examined and in the data set, by each one of the periodontal protocols (extent of periodontitis); Prevalence of Periodontitis (no, moderate and severe), based on different combinations of the assessed periodontal parameters.

Table I Characterization of periodontal protocols according to the evaluated teeth and sites

\begin{tabular}{|c|c|c|c|}
\hline Type of protocol & Selected teeth & $\mathbf{N}^{\circ}$ of teeth & Probing sites \\
\hline Full mouth (FMPE) & All teeth & 28 & $\begin{array}{l}\text { DB-B-MB-DL-L-ML* } \\
(n=168)\end{array}$ \\
\hline $\begin{array}{l}\text { Community Periodontal Index } \\
(\mathrm{CPI})^{\mathrm{a}}\end{array}$ & $\begin{array}{l}\text { Index teeth: I7/I6, II, 26/27, 36/37, 4I, } \\
46 / 47\end{array}$ & 10 & $\begin{array}{l}\text { All (circumferential) } \\
6 \text { records }\end{array}$ \\
\hline CPI Modified $(\mathrm{CPIm})^{\mathrm{b}}$ & All teeth & $28(\mathrm{PD}) * 10(\mathrm{CAL})$ & $\begin{array}{l}\text { All (circumferential) } \\
28 \text { PD records; } 6 \mathrm{CAL} \\
\text { records }\end{array}$ \\
\hline Full-mouth 3 (FM3) & All teeth & 28 & MB-B-DL $(n=84)$ \\
\hline Random Half-mouth 6 (HM6) & 2 random quadrants ${ }^{* *}$ & 14 & DB-B-MB-DL-L-ML $(n=84)$ \\
\hline Random Half-mouth 3 (HM3) & 2 random quadrants & 14 & MB-B-DL (n=42) \\
\hline
\end{tabular}

*PD, probing depth; CAL, clinical attachment level; DB, disto-buccal; B, buccal, MB, mesio-buccal; DL, disto-lingual; L, lingual; ML, mesio-lingual

**All teeth from two randomly selected quadrants (one maxillary and one mandibular)

aWorld Health Organization, 1997; 'World Health Organization, 2013

In the comparative analysis between CPI and CPIm, prevalence of periodontitis was based on a combination of scores that has been used for this index ${ }^{14}$ considering for moderate periodontitis the occurrence of at least one site with $\mathrm{PD} \geq 4 \mathrm{~mm}$ and one site with $\mathrm{CAL} \geq 4 \mathrm{~mm}$, and for severe periodontitis, one or more sites with $\mathrm{PD} \geq 4 \mathrm{~mm}$ and one or more sites with $\mathrm{CAL} \geq 4 \mathrm{~mm}$. In the comparative evaluation between CPIm and other PMPEs, in relation to FMPE, three case definitions were used:

1. At least one site with PD or CAL $\geq 4 \mathrm{~mm}^{7}$

2. At least one site with $\mathrm{PD} \geq 4 \mathrm{~mm}$ and one site with $\mathrm{CAL} \geq 4 \mathrm{~mm} .^{14}$

3. At least two sites with $\mathrm{PD} \geq 4 \mathrm{~mm}$ and two sites with $\mathrm{CAL} \geq 4 \mathrm{~mm}$ in different teeth.
Initially, a comparison was made between CPI and CPIm with regard to the prevalence of BOP, PD and CAL at levels of $4 \mathrm{~mm}$ and $6 \mathrm{~mm}$, and the prevalence of moderate and severe periodontitis, based on the Pearson's chi-square test and the values of sensitivity of both indices in relations to the FMPE. Due to the lower accuracy found for the CPI in relation to its new version, the CPIm, only the latter was included in the comparisons with the three PMPEs evaluated.

The comparison among prevalence rates was based on sensitivity, absolute bias, relative bias and ainflation factor, calculated as described by several studies, ${ }^{11,15,16}$ as follows:

$$
\text { Sensitivity }=\frac{\text { PMPE prevalence }}{\text { FMPE prevalence }} \times 100
$$


Absolute bias $=$ FMPE prevalence - PMPE prevalence

Relative bias $=\frac{\text { PMPE prevalence }- \text { FMPE prevalence }}{\text { FMPE prevalence }} \times 100$

$$
\text { Inflation factor }=\frac{\text { FMPE prevalence }}{\text { PMPE prevalence }}
$$

As the data for PMPEs were obtained from the FMPE, there was no possibility of occurrence of false positives, which results in a specificity calculation of 1 for all PMPE that is why this indicator was not presented in the results.

Statistical comparison of the prevalence rates estimated by the different examination protocols was made by Pearson's chi-square test; the Cochran's Q test was used for the comparisons among the different case definitions; the Wilcoxon test was used to compare mean frequencies of PD and CAL between the FMPE and each PMPE (severity and extent of periodontitis). In all analyzes, a level of statistical significance of $5 \%(\mathrm{p}<0.05)$ was considered. Statistical Package for the Social Sciences (SPSS) v.22 (IBM®) was used to process and analyze the data.

This study received approval from the Human Research Ethics Committee of the Menino Jesus Municipal Hospital, Sao Paulo (certificate number: 1.177.8730), and all participants signed a statement of informed consent.

\section{Results}

The total sample comprised 350 individuals, from 35 to 74 years (mean: $52.8 \pm 10.5$ years), and more than half of the participants (58\%) were younger than 55 years. The female sex predominated $(59.7 \%)$ and also the university education (45.4\%), followed by a high school education $(32.3 \%)$. More than half of the sample $(58.3 \%)$ had one to four missing teeth (mean: $5.4 \pm 9.0$ ), and about $65 \%$ of the participants presented all sites with $\mathrm{PD}<4 \mathrm{~mm}$ (data not shown in table).

The prevalence of BOP was $40.6 \%$, moderate periodontitis $22.0 \%$ and severe periodontitis $8.2 \%$, according to the FMPE. Table 2 presents a comparative evaluation of the periodontal parameters estimated by the CPI and the CPIm, in relation to the FMPE. CPI underestimated all parameters, presenting a sensitivity of $50 \%$ in estimating prevalence of periodontitis. CPIm was similar to FMPE to detect BOP and periodontal pocket, but it underestimated CAL by approximately $10 \%$, with sensitivity of $80 \%$ to estimate moderate and severe periodontitis, with greater accuracy than its previous version.

As shown in Table 3, significant differences were found in the estimates of prevalence of periodontitis among the different examination protocols and the different case definitions considered $(p<0.001)$. Absolute and relative biases demonstrated that all PMPE underestimated the prevalence of periodontitis for each of the case definition, with the relative bias ranging from -10.7 to $-54.7 \%$ in relation to the real prevalence (FMPE). CPIm and FM3 protocol presented the highest sensitivity in relation to FMPE $(p<0,05)$ and were similar to each other $(\mathrm{p}>0,05)$. The worst sensitivity was that of the HM3 protocol. Both the HM6 and HM3 protocols differed significantly from the CPIm. The inflation factor required for adjustment of prevalence rates was less than 1.5 for CPIm and for FM3 in any case definition, and for HM6 in definition 1. As the case definition was more rigorous in the criteria, there were significant reduction in the sensitivity of all PMPE, and greater inflation factor was required.

Table 2 Comparison of estimates of prevalence of bleeding on probing, periodontal pockets and attachment loss, according to full-mouth protocol examination (FMPE), Community Periodontal Index (CPI) and Community Periodontal Index modified (CPIm). Sao Paulo, 2016

\begin{tabular}{|c|c|c|c|c|c|c|c|c|}
\hline \multirow[t]{2}{*}{ Periodontal measures } & \multicolumn{2}{|c|}{ FMPE } & \multicolumn{3}{|c|}{ CPI } & \multicolumn{3}{|c|}{ CPIm } \\
\hline & $\mathbf{n}$ & $\%$ & $\mathbf{n}$ & $\%$ & sensitivity & $\mathbf{n}$ & $\%$ & sensitivity \\
\hline Bleeding on probing & 142 & 40.6 & 106 & $30,3^{*}$ & 0.7 & 142 & 40.6 & 1.0 \\
\hline \multicolumn{9}{|l|}{ Probing depth } \\
\hline $\mathrm{PD} \geq 4 \mathrm{~mm}$ & 94 & 26.8 & 63 & $18.0 *$ & 0.7 & 94 & 26.8 & 1.0 \\
\hline $\mathrm{PD} \geq 6 \mathrm{~mm}$ & 28 & 8.3 & 19 & $5,4^{*}$ & 0,7 & 28 & 8.3 & 1.0 \\
\hline \multicolumn{9}{|l|}{ Attachment loss } \\
\hline $\mathrm{CAL} \geq 4 \mathrm{~mm}$ & 122 & 34.8 & 91 & $26^{*}$ & 0.7 & 91 & $26.0 *$ & 0.7 \\
\hline $\mathrm{CAL} \geq 6 \mathrm{~mm}$ & 38 & 10.8 & 27 & $7,7^{*}$ & 0.7 & 27 & $7.7 *$ & 0.7 \\
\hline Moderate periodontitis ${ }^{a}$ & 77 & 22.0 & 40 & $11.4^{*}$ & 0.5 & 63 & $18.0 *$ & 0.8 \\
\hline Severe periodontitis ${ }^{\mathrm{b}}$ & 31 & 8.8 & 16 & $4,2^{*}$ & 0.5 & 25 & $7.1 *$ & 0.8 \\
\hline
\end{tabular}

$*_{p}<0,05$ (Pearson's Chi-square test)

at least one tooth with PD $\geq 4 \mathrm{~mm}$ and one tooth with $C A L \geq 4 \mathrm{~mm}$

bat least one tooth with PD $\geq 4 \mathrm{~mm}$ and one tooth with $C A L \geq 6 \mathrm{~mm}$ 
Table 3 Comparison of estimates of periodontitis prevalence based on three case definitions according to full-mouth and partial-mouth periodontal examination protocols. Sao Paulo, 2016

\begin{tabular}{|c|c|c|c|c|c|}
\hline & FMPE & CPIm & FM3 & HM6 & HM3 \\
\hline \multicolumn{6}{|l|}{ Definition Ia } \\
\hline Prevalence & $122(34.8 \%)$ & 109 (3I.I\%) & 107 (30.6\%) & $95(27.1 \%)^{*}$ & $78(22.4 \%)^{*}$ \\
\hline Sensitivity (\%) & & 89.3 & 87.8 & 77.8 & 63.9 \\
\hline Absolute bias & & 13 & 15 & 27 & 44 \\
\hline Relative bias (\%) & & -10.7 & -12.2 & -22.2 & -36.1 \\
\hline Correction factor & & I.I & I.I & 1.3 & 1.6 \\
\hline \multicolumn{6}{|l|}{ Definition $2^{b}$} \\
\hline Prevalence & 77 (22.0\%) & $63(18.0 \%)$ & $62(17.7 \%)$ & $50(14.3 \%)^{*}$ & $38(10.8 \%)^{*}$ \\
\hline Sensitivity (\%) & & 81.8 & 80.5 & 64.9 & 49.3 \\
\hline Absolute bias & & 14 & 15 & 27 & 39 \\
\hline Relative bias (\%) & & -18.2 & -19.5 & -35.1 & -50.7 \\
\hline Correction factor & & 1.2 & 1.2 & 1.5 & 2.0 \\
\hline \multicolumn{6}{|l|}{ Definition $3^{c}$} \\
\hline Prevalence & $66(18.8 \%)$ & 48 (I3.7\%) & 46 (13.1\%) & $37(10.6 \%)^{*}$ & $29(8.3 \%)^{*}$ \\
\hline Sensitivity (\%) & & 72.7 & 69.7 & 57.8 & 45.3 \\
\hline Absolute bias & & 18 & 20 & 27 & 35 \\
\hline Relative bias (\%) & & -37.3 & -30.3 & -42.2 & -54.7 \\
\hline Correction factor & & $\mathrm{I} .4$ & 1.5 & 1.7 & 2.2 \\
\hline
\end{tabular}

$\mathrm{p}<0.00$ I between full-mouth protocol and all partial protocols (Pearson's chi-square test) and among definitions (Cochran's $\mathrm{Q}$ test); ${ }^{\mathrm{p}}<0,00 \mathrm{I}$ in relation to CPIm (Pearson's chi-square test)

FMPE, Full-mouth protocol examination; CPIm, Community Periodontal Index modified (WHO, 20I3); FM3, Full-mouth three (MB, B, DL sites in all teeth); HM6, Half-mouth six (six sites in 2 random quadrants); HM3, Half-mouth three sites (MB, B and DL sites in 2 random quadrants)

at least one site with $\mathrm{PD} \geq 4 \mathrm{~mm}$ OR one site with $\mathrm{CAL} \geq 4 \mathrm{~mm}$

bat least one site with PD $\geq 4 \mathrm{~mm}$ AND one site with $C A L \geq 4 \mathrm{~mm}$

cat least two or more sites with PD $\geq 4 \mathrm{~mm}$ AND two or more sites with CAL $\geq 4 \mathrm{~mm}$;

The participants presented on average $6.2 \%$ of teeth with BOP in at least one site (Table 4). BOP frequencies were similar among FMPE, CPIm and FM3, but were underestimated by HM6 and HM3 $(\mathrm{p}<0.05)$. Mean PD and mean CAL in the sample were less than $1.5 \mathrm{~mm}$. In the comparative evaluation of periodontitis severity estimates by M-PD and M-CAL, the FM3 and HM6 protocols were similar to FMPE ( $>00.05)$; CPIm and FM3 differed significantly from FMPE ( $<<0.01)$, and the CPIm overestimated M-PD by $31.8 \%$ and M-CAL by $66.6 \%$. Proportion of affected periodontal sites was low in the sample, and about $95 \%$ of the evaluated sites had PD and CAL lower than $4 \mathrm{~mm}$. Both site and tooth ratios at the three PD and CAL levels were similar among FMPE, FM3 and HM6 protocols, but underestimated by the HM3 protocol $(p<0.01)$. The CPIm does not apply for estimates of extent of affected sites because the index records only one site per tooth. Regarding to the proportion of affected teeth, the CPIm was similar to FMPE in periodontal pockets extension, but overestimated $\mathrm{CAL} \geq 4 \mathrm{~mm}$ and $\geq 6 \mathrm{~mm}(\mathrm{p}<0.01)$.

\section{Discussion}

This study made a comparative evaluation of three partialmouth periodontal examination protocols recently recommended for epidemiological surveys ${ }^{8}$ and of the last two versions of the Community Periodontal Index (CPI and CPI modified), from WHO, ${ }^{9,12}$ which were not yet investigated together. The findings showed the superiority of the modified CPI in relation to its previous version. It was similar to the FMPE for estimates of bleeding on probing and periodontal pockets and showed $80 \%$ sensitivity to estimate prevalence of moderate and severe periodontitis, contrasting with $50 \%$ in the previous CPI

The best performance of CPIm was probably due to the assessment of probing depth on all the present teeth. However, CAL continues to be assessed on 10 index teeth, which makes the two versions similar regarding this variable. If the greatest measure of CAL on each tooth was recorded as for PD, its sensitivity would be $100 \%$. 
Table 4 Comparison of extent and severity of periodontitis based on mean frequency of the assessed periodontal parameters according to full-mouth and partial-mouth periodontal examination protocols. Sao Paulo, 2016

\begin{tabular}{|c|c|c|c|c|c|}
\hline Variable & FMPE & CPIm & FM3 & HM6 & HM3 \\
\hline $\begin{array}{l}\text { Bleeding on probing } \\
(\% \text { teeth } \pm S D)\end{array}$ & $6.2 \pm 12.0$ & $6.2 \pm 12.0$ & $5.9 \pm 10.0$ & $4.2 \pm 6.1$ & $3.9 \pm 8.2$ \\
\hline \multicolumn{6}{|c|}{ Probing depth $(X \pm S D)$} \\
\hline Mean PD (mm) & $1.16 \pm 0.38$ & $1.35 \pm 0.56$ & $1.18 \pm 0.37$ & $1.16 \pm 0.38$ & I. $18 \pm 0.42$ \\
\hline$p^{*}$ & ref. & $<0.01$ & 0.01 & 0.13 & 0.03 \\
\hline$\%$ sites $\mathrm{PD}<4 \mathrm{~mm}$ & $96,9 \pm 11,5$ & NA & $97.1 \pm 10.5$ & $97.0 \pm 13.19$ & $97.5 \pm 12.23$ \\
\hline$\%$ sites $\mathrm{PD} \geq 4 \mathrm{~mm}$ & $3,2 \pm 8,7$ & NA & $2.9 \pm 8.2$ & $3.0 \pm I I .1$ & $2.5 \pm 5.4$ \\
\hline$\%$ sites $P D \geq 6 m m$ & $0.7 \pm 3,3$ & NA & $0.6 \pm 3.2$ & $0.6 \pm 3.7$ & $0.5 \pm 4.3$ \\
\hline$p^{*}$ & ref. & & 0.42 & 0.77 & 0.04 \\
\hline$\%$ teeth $\mathrm{PD}<4 \mathrm{~mm}$ & $95,2 \pm 12,4$ & $95.2 \pm 12.4$ & $95.7 \pm 10.4$ & $95.8 \pm 10.7$ & $97.0 \pm 12.23$ \\
\hline$\%$ teeth $P D \geq 4 m m$ & $4,8 \pm 10,3$ & $4.8 \pm 10.3$ & $4.3 \pm I I .1$ & $4.2 \pm 10.1$ & $3.0 \pm 8.9$ \\
\hline$\%$ teeth $P D \geq 6 \mathrm{~mm}$ & $\mathrm{I}, 0 \pm 7,5$ & $1.0 \pm 8.2$ & $0.8 \pm 2.4$ & $0.8 \pm 3.4$ & $0.4 \pm 3.6$ \\
\hline $\mathrm{P}^{*}$ & ref. & 1.0 & 0.77 & 0.65 & 0.25 \\
\hline \multicolumn{6}{|c|}{ Attachment loss $(\mathrm{X} \pm \mathrm{SD})$} \\
\hline Mean CAL (mm) & $1.38 \pm 0.49$ & $1.53 \pm 0.66$ & $1.37 \pm 0.48$ & $1.38 \pm 0.49$ & $1.37 \pm 0.47$ \\
\hline $\mathrm{P}^{*}$ & & $<0.01$ & $<0.01$ & 0.47 & 0.54 \\
\hline$\%$ sites $C A L<4 m m$ & $95,9 \pm 5,5$ & NA & $96.2 \pm 11.2$ & $96.4 \pm 12.6$ & $97.1 \pm 10.9$ \\
\hline$\%$ sites $C A L \geq 4 m m$ & $4, I \pm 8,8$ & NA & $3.8 \pm 8.5$ & $3.6 \pm 9.4$ & $2.9 \pm 9.1$ \\
\hline$\%$ sites $C A L \geq 6 \mathrm{~mm}$ & $0,9 \pm 3,8$ & NA & $0.8 \pm 3.3$ & $0.7 \pm 3.9$ & $0.6 \pm 3.7$ \\
\hline $\mathrm{P}^{*}$ & ref. & & 0.78 & 0.25 & 0.02 \\
\hline$\%$ teeth $C A L<4 m m$ & $94,0 \pm 12,4$ & $92.4 \pm 13.4$ & $94.2 \pm 11.3$ & $I, 0 \pm 2.1$ & $96.3 \pm 10.9$ \\
\hline$\%$ teeth $C A L \geq 4 m m$ & $6,0 \pm 11,4$ & $7.6 \pm 11.1$ & $4.4 \pm 8.6$ & $4.2 \pm 9.6$ & $3.7 \pm 9.0$ \\
\hline$\%$ teeth $C A L \geq 6 \mathrm{~mm}$ & $\mathrm{I}, 3 \pm 4,5$ & $1.8 \pm 7.5$ & $1.4 \pm 6.2$ & $1.3 \pm 6.2$ & $0.8 \pm 4.2$ \\
\hline $\mathrm{P}^{*}$ & ref. & $<0,0$ l & 0.95 & 0.06 & $<0.01$ \\
\hline
\end{tabular}

FMPE, full-mouth protocol examination; CPIm, community periodontal index modified (WHO, 20I3); FM3, full-mouth three sites (MB, B, DL sites in all teeth); HM6, half- mouth six sites (six sites in 2 random quadrants); HM3, half-mouth three sites (MB, B and DL sites in 2 random quadrants)

Total of probing sites: $F M P E=168 ; F M 3$ and $H M 6=84 ; \mathrm{HM} 3=42$

Total of probing teeth: FMPE=28; $C P I m=28$ (PD) and 10 (CAL); FM3 and HM6=28; HM3 $=14$

*Wilcoxon test between FMPE and each partial protocol considering all cases with $\mathrm{PD} / \mathrm{CAL} \geq 4 \mathrm{~mm}$ together.

All the PMPE protocols underestimated prevalence of periodontitis when compared to the FMPE for all case definitions employed, with high relative biases. The CPIm exhibited the best sensitivity and minor bias, which was similar to that found for the FM3 protocol $(\mathrm{p}>0.05)$. Both methods involve the examination of all teeth, differing in the number of sites examined and recorded. The protocols involving only two quadrants (HM6 and HM3) had significantly higher biases in comparison to CPIm and FM3, although previous studies report good levels of accuracy with these protocols. ${ }^{5,7,16}$ This divergence may be due to the low severity and extent of periodontitis in the present sample, as $65.7 \%$ of the participants had all sites evaluated with PD/ CAL $<4 \mathrm{~mm}$ and less than $5 \%$ of the teeth per individual had altered periodontal variables. These rates are much lower than those reported in other studies. ${ }^{10,17,18}$ The low frequency of affected teeth must have exerted an influence on the detection of cases, especially when using protocols in which fewer sites were examined. The influence of the level of periodontitis in the population on the sensitivity of a PMPE protocol has been described previously, as well as the case definition. $5,7,18$

In the present study, three case definitions were considered in increasing order of criterion requirements, which exhibited significant differences according to the PMPE used. These findings are in agreement with data described by Eke et al., ${ }^{19}$ who reported relative biases ranging from $29.0 \%$ to $70.0 \%$ when comparing the prevalence estimates by PMPE protocols used in official surveys in the USA 
based on different case definitions. The more altered parameters are required to define a case, the greater biases in PMPE estimates, which is due to the number of sites examined.

Although there has been a lot of discussion and considerable controversy about a standard case definition, a definition proposed by the Centers for Disease Control and Prevention and American Academy of Periodontology (CDC/AAP) has been recommended. ${ }^{8,20}$ In the USA, it has been adopted, along with the FMPE, for the updating of official epidemiological data on periodontal disease. ${ }^{21,22}$ In the present study it was not used because this is a case definition with quite strict criteria wichlimitateits use withpartial protocols. With regard to CPI/CPIm, proximal sites are not identifiable; in PMPEs, the use of only proximal sites considerably reduces the number of registered sites, which would possibly result in greater bias.

In an attempt to minimize bias, some studies have proposed a reduction in the criteria required for the definition of a case proportionally to the number of sites examined when a PMPE protocol is used. ${ }^{6,11}$ Although there is no standardization for that conduct, the differences found in this study regarding the three hypothetical case definitions employed has shown that relation. The inflation factor is another means of adjustment for prevalence rates estimated by PMPE protocols ${ }^{5,14}$ and this study showed the increase in that factor with the increase of a criterion in the case definition, as well as with the reduction in the number of sites/teeth examined.

Although accuracy of PMPE protocols in estimating periodontitis prevalence has been widely investigated in the literature, biases related to the estimates of severity and extent of the disease when using partial protocols have been little evaluated. ${ }^{7}$ In the present study, mean PD, mean CAL and proportions of affected sites were evaluated. These aspects varied little in the majority of PMPE protocols and were not influenced by the case definition, unlike what occurred with prevalence of the disease. These findings are in agreement with data described by Vettore et al. ${ }^{23}$ and Kingman et al. ${ }^{5}$ who found low bias in estimates of severity and extension of periodontal it is by PMPEs.

The CPIm exhibited good sensitivity regarding the estimate of periodontit is prevalence, although this index had the highest biases regarding the estimates of M-PD (>30\%) and M-CAL (>60\%) and it overestimated the extent of CAL $\geq 4 \mathrm{~mm}$ by approximately $30 \%$. The high levels of overestimates by CPIm for these variables may be explained by the fact that this protocol only records the highest parameter of each tooth, as the means are calculated based on the sum of the scores and the total number of sites examined. Thus, the use of CPIm for estimating proportion of affected sites per individual as well as M-PD and M-CAL is considered unviable. Therefore, regarding severity and extent of periodontitis, the PMPEs were better than the CPIm, with the FM6 protocol achieving similar results to the FMPE and the other protocols exhibiting low levels of bias, which is in agreement with other studies. ${ }^{5,23}$

The present study refers to a specific population in which the occurrence and severity of periodontitis were relatively low, being its main limitation. Thus, the validity of the findings needs to be confirmed in population-based studies. As the characteristics of populations with varied levels of disease severity constitute an important aspect of the performance of partial protocols, a good measure would be to perform a complete periodontal examination on a random subsample of the population to be evaluated to obtain direct evidence of the probable magnitude of the biases incurred when a given PMPE is used in this population. Thus, the calculation of an inflation factor could bring the estimates closer to the real parameters.

\section{Conclusion}

The CPIm and the full-mouth 3 sites protocol presented satisfactory sensitivity to estimate prevalence of periodontitis in populations, being superior to the previous CPI and to the random half-mouth protocols. However, accuracy of estimates may vary with the case definition and population characteristics.

\section{Funding}

CAPES (Brazil)- Coordenação de Aperfeiçoamento de Pessoal de Nível Superior.

\section{Acknowledgments}

Authors thank the Advento Study Group, and the coleagues Borreli L, Contrera LMO and Rizzioli AKF for their collaboration in data collection.

\section{Conflict of interest}

The authors declares that there is no conflict of interest.

\section{References}

1. Meusel DRDZ, Ramacciato JC, Motta RHLM, et al. Impact of the severity of chronic periodontal disease on quality of life. Journal of Oral Science. 2015;57(2):87-94

2. Petersen PE, Ogawa H. The global burden of periodontal disease: towards integration with chronic disease prevention and control. Periodontology 2000. 2012;60(1):15-39.

3. Leroy R, Eaton KA, Savage A. Methodological issues in epidemiological studies of periodontitis - how can it be improved? BMC Oral Health. 2010;10:8.

4. Natto ZS, Ahmad RHA, Alsharif LT, et al. Chronic Periodontitis Case Definitions and Confounders in Periodontal Research: A Systematic Assessment. BioMed Research International. 2018;9.

5. Kingman A, Susin C, Albandar JM. Effect of parcial recording protocols on severity estimates of periodontal disease. J Clin Periodontol. 2008;35(8):659-667.

6. Heaton B, Sharma P, Garcia RI, et al. Evaluating periodontal disease misclassification mechanisms under partial-mouth recording protocols. J Clin Periodontol. 2018;45(4):422-430.

7. Tran DT, Gay I, Du XL, et al. Assessing periodontitis in populations: a systematic review of validity of parcial-mouth examination protocols. $J$ Clin Periodontol. 2013;40(12):1064-1071.

8. Holtfreter B, Albandar JM, Dietrich T, et al. Standards for reporting chronic periodontitis prevalence and severity in epidemiologic studies. Proposal standards from the joint EU/USA Periodontal Epidemiology Working Group. J Clin Periodontol. 2015;42(5):407-412.

9. World Health Organization. Oral Health surveys: Basic methods. $4^{\text {th }}$ ed. England: World Health Organization; Geneva: 1997. p. 36-39.

10. Peres MA, Peres KG, Cascaes AM, et al. Validity of Partial Protocols to assess the prevalence of periodontal outcomes and associated sociodemographic and behavior factors in adolescents and young adults. J Periodontol. 2012; 83(3):369-378. 
11. Tran DT, Gay I, Du XL, et al. Assessment of parcial-mouth periodontal examination protocols for periodontitis surveillance. J Clin Periodontol. 2014;41(9):846-852.

12. World Health Organization. Oral Health surveys: Basic methods. $5^{\text {th }}$ ed. England: World Health Organization; Geneva: 2013. p. 47-51.

13. Teixeira FCF, Marin-Leon L, Gomes EP, et al. Relationship between periodontitis and subclinical risk indicators for chronic noncommunicablediseases. Braz. Oral Res. 2020;34e058.

14. Lorenzo SA. Periodontal conditions and associated factors among adults and the elderly: findings from the first National Oral Health Survey in Uruguay.Cad. SaúdePública. 2015;31(11):2425-2436.

15. Kingman A, Albandar JM. Methodological aspects of epidemiological studies of periodontal diseases. Periodontology 2000. 2002;29(1):11-30.

16. Susin C, Kingman A, Albandar JM. Effect of partial recording protocols on estimates of prevalence of periodontal disease. J Periodontol. 2005;76(2):262-267.

17. Susin C, Vecchia CFD, Opperman RV, et al. Periodontal attachment loss in an urban population of Brazilian adults: effect of demographic, behavioral and environment risk indicators. $J$ Periodontol. 2004;75(7):1033-1041.
18. Eke PI, Dye L, Wei GO, et al. Prevalence of periodontitis in Adults in United States: 2009 and 2010. J Dent Res. 2012;91(10):914-920.

19. Eke PI, Thorton-Evans GO, Wei L, et al. Accuracy of NHANES periodontal examination protocols. J Dent Res. 2010;89(11):1208-1213.

20. Tonetti MS, Greenwell H, Kornman KS. Staging and grading of periodontitis: framework andproposalof a new classificationand case definition. J ClinPeriodontol. 2018;45(20 Suppl):S149-S161.

21. Eke PI, Page RC, Wei, L, et al. Update of case definitions for population-based surveillance of periodontitis. $J$ Periodontol. 2012;83(12):1445-1454.

22. Eke. Risk factors for periodontitis in US adults: NHANES 2009 to 2012. J Periodontol. 2016; 87(10):1174-1184.

23. Vettore MV, Lamarca G, Leão AT, et al. Parcial recording protocols for periodontal disease assessment in epidemiological surveys. CadSaude Publica. 2007;23(1):33-42. 